\title{
Catarata pediátrica pós-trauma
}

\author{
Traumatic pediatric cataract
}

\author{
Fernando Antonio de M. Leal ${ }^{1}$ \\ Márcia Beatriz Tartarella ${ }^{2}$
}

Universidade Federal de São Paulo (UNIFESP)/Escola Paulista de Medicina (EPM)

${ }^{1}$ Doutorando em oftalmologia pela UNIFESP/EPM.

Doutora em oftalmologia pela UNIFESP/EPM. Chefe do Serviço de Catarata Congênita da UNIFESP/EPM.

Endereço para correspondência: Fernando Antonio M. Leal - Rua Maestro Cardim n 508 Apt 317 - São Paulo (SP) CEP 01323-000

E-mail:faml@terra.com.br

Recebido para publicação em 11.04.2003

Versão revisada recebida em 12.11.2003

Aprovação em 14.11.2003

Nota Editorial: Pela análise deste trabalho e por sua anuência na divulgação desta nota, agradecemos aos Drs. Liana Maria V. de Oliveira Ventura e Fábio Henrique Cacho Casanova.

\begin{tabular}{|c|}
\hline RESUMO \\
\hline $\begin{array}{l}\text { Objetivo: Estudar a catarata pediátrica pós-trauma, com relação ao tipo de } \\
\text { trauma, o tempo decorrido entre este e a cirurgia e a correlação entre a } \\
\text { acuidade visual obtida como tratamento realizado. Métodos: Estudo retros- } \\
\text { pectivo de pacientes atendidos no serviço de Catarata Congênita da } \\
\text { Universidade Federal de São Paulo, no período de agosto de } 1988 \text { a dezembro } \\
\text { de } 2001 \text {, com diagnóstico de catarata pediátrica pós-trauma. Resultados: } \\
\text { Foram revisados } 66 \text { prontuários de pacientes com diagnóstico de catarata } \\
\text { pediátrica após trauma, correspondendo a uma incidência de } 4,80 \% \text { do total } \\
\text { de casos atendidos no serviço. Com relação ao sexo, } 47(71,22 \%) \text { eram do sexo } \\
\text { masculino e } 19(28,78 \%) \text { do sexo feminino. Do total de casos de trauma, } 35 \\
\text { (53,03\%) foram contusos, } 21 \text { ( } 31,82 \%) \text { penetrantes e } 10(15,15 \%) \text { nãoclassi- } \\
\text { ficados. O tempo médio decorrido entre o trauma e a cirurgia foi de } 7 \text { anos } \\
\text { e seis meses. As principais complicações pós-operatórias descritas foram } \\
\text { seqüelas de uveítes em } 13 \text { pacientes }(21,12 \%) \text { e opacificação de cápsula } \\
\text { posterior em } 10 \text { (15,15\%). Em } 30 \text { olhos foi possível obtermos as acuidades } \\
\text { visuais inicial e final; deste total, } 1 \text { olho }(3,33 \%) \text { possuía AV inicial superior } \\
\text { ou igual a } 20 / 60 \text { com melhor correçãoe } 12(40 \%) \text { olhos AV final superior ou } \\
\text { igual a } 20 / 60 \text { com melhor correção. Os pacientes foram acompanhados em } \\
\text { média por } 2 \text { anos. Conclusão: A melhora daacuidade visual foi estatisticamen- } \\
\text { te significante (teste de Wilcoxon p<0,001), sendo mais intensa nos olhos } \\
\text { submetidos a tratamento cirúrgico (p<000,1) do que nos olhos submetidos } \\
\text { a tratamento clínico (p=0,043). }\end{array}$ \\
\hline
\end{tabular}

Descritores: Extração de catarata; Catarata/patologia; Acuidade visual; Ferimentos e lesões; Ferimentos penetrantes; Pseudofacia

\section{INTRODUÇÃO}

O trauma ocular é a principal causa de cegueira unilateral não-congênita em pacientes com menos de 20 anos de idade ${ }^{(1)}$. O tratamento da catarata resultante de traumas tem sofrido importantes modificações nas últimas duas décadas, decorrentes do desenvolvimento de novas técnicas cirúrgicas, melhor correção da afacia e melhor conhecimento do processo ambliogênico $^{(2)}$.

Até o início da década de 1980, a correção da afacia com o uso de lentes de contato era a alternativa de escolha ${ }^{(3)}$. O desenvolvimento das técnicas microcirúrgicas, introdução de materiais biocompatíveis e novos modelos de lentes intra-oculares levaram a uma importante melhora dos resultados visuais, entretanto, seguidas no início por inúmeras complicações ${ }^{(2)}$. Em catarata pediátrica as maiores complicações pós-operatórias são: uveíte fibrinosa, opacificação de cápsula posterior e ambliopia ${ }^{(4-5)}$.

Atualmente, o implante de lentes intra-oculares seguido por uma severa terapia anti-ambliogênica tem trazido melhores resultados visuais no tratamento da catarata pediátrica após trauma. Estudos relatam que 64,7\% a 80\% 
dos pacientes atingem uma visão superior ou igual a $20 / 60^{(6-8)}$.

O objetivo deste trabalho é estudar a catarata pediátrica pós-trauma, com relação ao tipo de trauma ocorrido, o tempo decorrido entre este e a cirurgia, correlacionando a melhora de acuidade visual com o tratamento realizado.

\section{MÉTODOS}

Foi realizado um estudo retrospectivo de pacientes atendidos no serviço de Catarata Congênita de Escola Paulista de Medicina / Universidade Federal de São Paulo no período de agosto de 1988 a dezembro de 2001 com diagnóstico de catarata pediátrica após trauma. Todos os casos referem-se a pacientes atendidos eletivamente, tendo sido a cirurgia primária, nos casos de traumas perfurantes, realizada em outros serviços de emergência oftalmológica. Os seguintes dados foram coletados: idade, gênero, olho acometido, data e tipo do trauma, tipo de cirurgia realizada, complicações pós-operatórias, acuidades visuais inicial e final e tempo de seguimento dos pacientes. A correlação entre acuidade visual inicial e final pós-tratamento realizado no serviço foi analisada através do teste de Wilcoxon.

\section{RESULTADOS}

Foram revisados 77 prontuários com diagnóstico de catarata pediátrica traumática o que corresponde a uma incidência de 5,44\% do total de pacientes atendidos no serviço. Destes prontuários, 66 tinham dados adequados para análise, correspondendo a uma incidência de 4,66\%. Com relação ao sexo, 47 pacientes $(71,21 \%)$ eram do sexo masculino e $19(28,78 \%)$ do sexo feminino. O Olho direto foi acometido em 34 pacientes $(51,52 \%)$ e o olho esquerdo em $32(48,48 \%)$. Os pacientes foram vítimas do trauma em média aos cinco anos e dois meses, tendo sido submetidos à cirurgia no serviço em média aos doze anos e oito meses (Gráfico 1). Em 35 pacientes $(53,03 \%)$ o trauma foi contuso, perfurante em $21(31,82 \%)$ e não classificado em $10(15,15 \%)$ casos (Gráfico 2).

Em relação ao procedimento cirúrgico realizado, 2 pacientes $(3,03 \%)$ foram submetidos à facoemulsificação com implante de lente intra-ocular, $17(25,75 \%)$ à facectomia extra-capsular com implante de lente intraocular, $4(6,06 \%)$ à facectomia extra-capsular sem implante de lente intra-ocular, $2(3,03 \%)$ ao implante secundário de lente intra-ocular, 6 pacientes $(9,09 \%)$ a outras cirurgias sem implante de lente intra-ocular, sendo duas lensectomias, duas lensectomias com vitrectomia anterior, uma iridectomia e uma vitrectomia anterior e 35 pacientes $(53,03 \%)$ não foram submetidos à cirurgia (Gráfico 3). As principais complicações pós-operatórias ocorridas foram sequielas de uveítes em 13 pacientes $(21,12 \%)$ e opacificação de cápsula posterior em 10 pacientes $(15,15 \%)$ (Gráfico 4). Os pacientes foram acompanhados em média por dois anos.

Em 30 olhos foi possível obtermos as acuidades visuais inicial e final. Deste total, um olho $(3,33 \%)$ possuía acuidade visual inicial superior ou igual a 20/60 com melhor correção e
$12(40,00 \%)$ olhos acuidade visual final superior ou igual a 20/60 com melhor correção (Gráfico 5). O teste de Wilcoxon, utilizado para verificar a significância de melhora da acuidade visual, apresentou nível de significância $\mathrm{p}=0,0043$ para 9 olhos submetidos ao tratamento clínico e $\mathrm{p}<0,001$ para 20 olhos submetidos a tratamento cirúrgico.

\section{DISCUSSÃO}

Do total de pacientes atendidos no serviço, 4,66\% eram portadores de catarata pós-trauma, sendo 47 pacientes

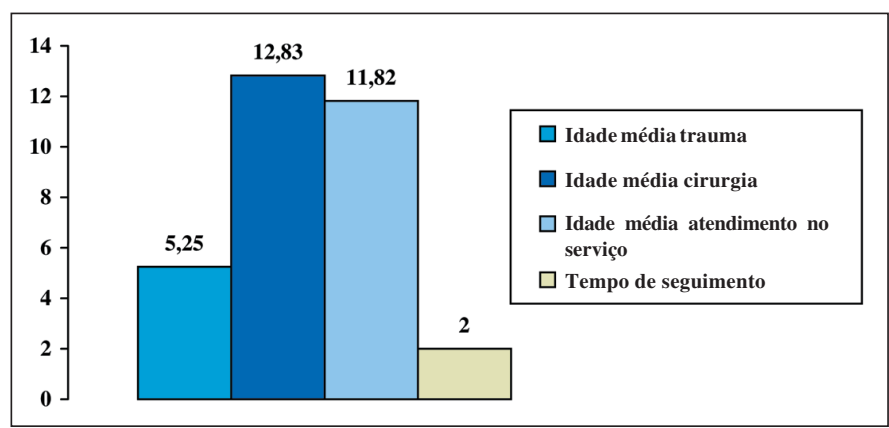

Gráfico 1 - Idade média dos pacientes (anos) com catarata pediátrica no momento do trauma, cirurgia, atendimento no serviço e tempo de seguimento dos mesmos

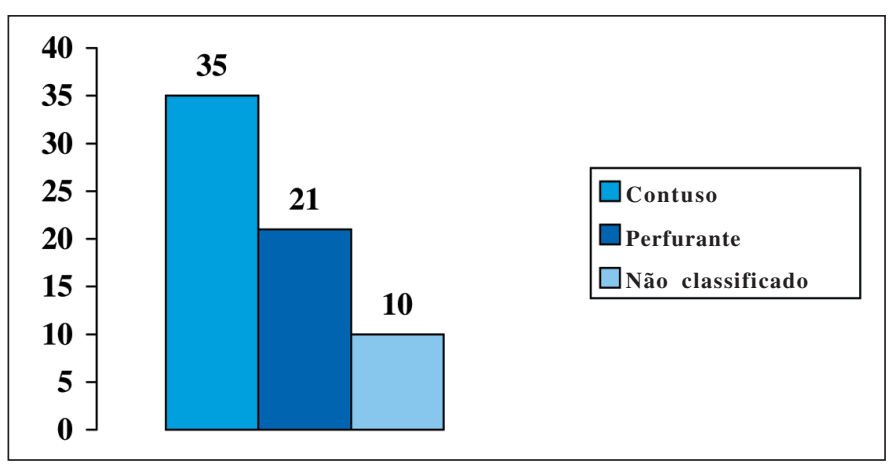

Gráfico 2 - Tipos de trauma em pacientes com catarata pediátrica

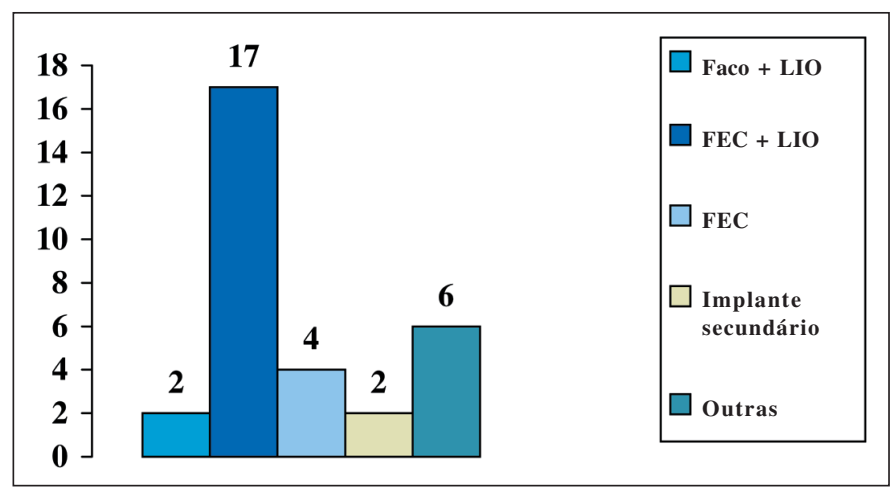

Gráfico 3 - Conduta cirúrgica realizada em pacientes com catarata pediátrica pós-trauma 


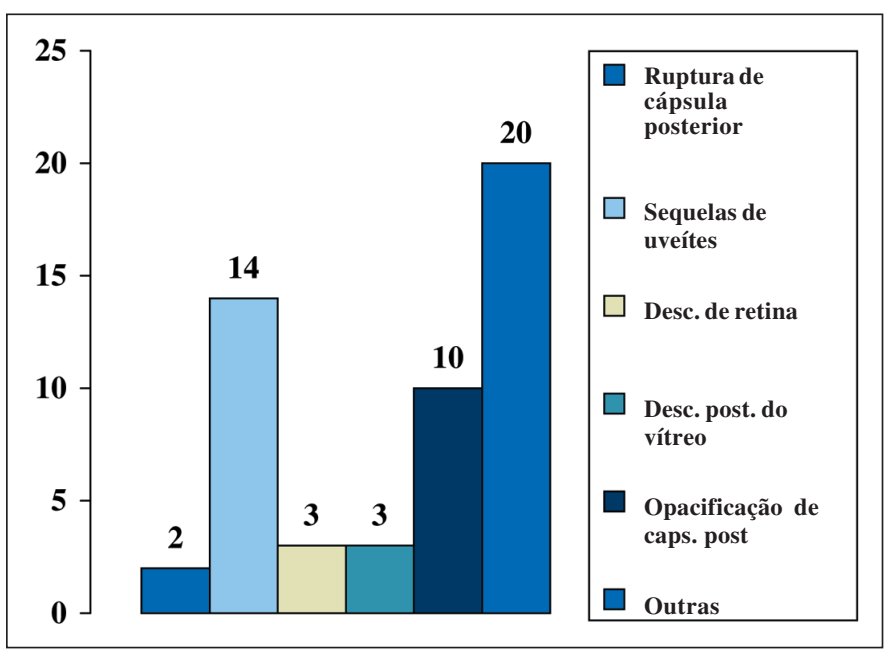

Gráfico 4 - Complicações pós-operatórias em pacientes com catarata pediátrica pós-trauma

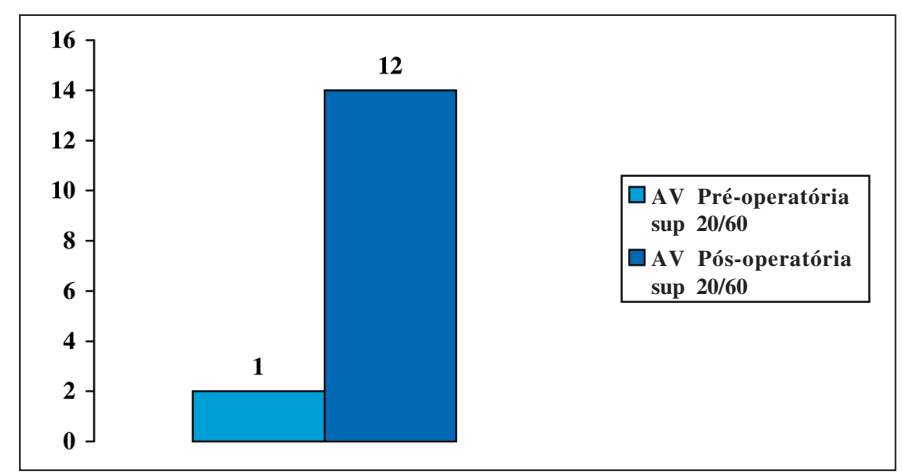

Gráfico 5 - Acuidade visual pré e pós-operatória em pacientes com catarata pediátrica pós-trauma

$(71,21 \%)$ do sexo masculino e $19(28,78 \%)$ do sexo feminino. Os dados estão de acordo com a literatura que relata taxas de incidência no sexo masculino variando de $78,84 \%$ a $85 \%$ e no sexo feminino de $15 \%$ a $21,15 \%{ }^{(2,7)}$. O olho direito foi acometido em 34 pacientes $(51,52 \%)$ e o olho esquerdo em $32(48,48 \%)$.

O trauma foi contuso em 35 pacientes $(53,03 \%)$ e perfurante em $21(31,81 \%)$. Estudos relatam uma incidência de $90 \%$ de traumas perfurantes e $10 \%$ de contusos em crianças portadoras de catarata traumática ${ }^{(2)}$ e uma incidência de $55 \%$ de traumas contusos e $45 \%$ de traumas perfurantes, encontrando excelentes resultados visuais (acuidade visual $>20 / 40$ ) em $86 \%$ de olhos com catarata traumática após trauma contuso, quando comparado com $38 \%$ de olhos com trauma penetrante. A melhor acuidade visual final em olhos com trauma contuso é atribuída à manutenção do eixo visual claro, colocação de lente intra-ocular no saco capsular e menor incidência de complicações pós-operatórias ${ }^{(4)}$.

Os pacientes foram vítimas do trauma em média aos 5 anos e 2 meses, tendo sido submetidos à cirurgia no serviço em média aos 12 anos e 8 meses. Em um estudo realizado sobre implante de lentes intra-oculares em crianças portadoras de catarata traumática na África do Sul, os melhores resultados visuais obtidos ocorreram em crianças que tinham mais de cinco anos de idade no momento do trauma, e cujo tempo decorrido entre este e a cirurgia de catarata foi inferior a um ano ${ }^{(8)}$.

Churchill e cols, em um trabalho sobre fatores que afetam o resultado visual em crianças após catarata traumática unilateral, mostraram que o retardo na realização da cirurgia pode afetar adversamente o resultado visual final ${ }^{(9)}$ A literatura relata que os resultados cirúrgicos em adultos que foram vítimas de trauma durante a infância, sem anormalidades do segmento posterior e ambliopia, e submetidos à cirurgia mesmo muitos anos após o trauma, que neste referido estudo variou entre 28 a 81 anos após o trauma inicial, é relativamente segura e freqüentemente resulta em uma melhora importante da acuidade visual ${ }^{(5)}$.

As principais complicações pós-operatórias observadas foram seqüela de uveítes em 20 pacientes $(21,12 \%)$ e opacificação de cápsula posterior em 10 pacientes $(15,15 \%)$. Trabalhos relatam incidências de uveítes variando de $19 \%$ a $61,61 \%$ e de opacificação de cápsula posterior variando de $83,36 \%$ a $100 \%$ dos $\operatorname{casos}^{(4,7)}$.

Em trinta olhos, no qual foi possível obtermos as acuidades visuais inicial e final, a melhora ocorrida foi estatisticamente significante $(\mathrm{p}<0,001)$, sendo que $40 \%$ dos olhos apresentaram acuidade visual final superior ou igual a 20/60 com melhor correção. No entanto, o nível de significância foi maior para os olhos submetidos ao tratamento cirúrgico $(\mathrm{p}<0,001)$ quando comparados com os olhos submetidos ao tratamento clínico $(\mathrm{p}=0,043)$. Diversos trabalhos na literatura apontam resultados visuais satisfatórios com implante de lente intra-ocular no tratamento da catarata traumática em crianças s, $^{(2,-6,8-12)}$.

O implante de LIO de câmara posterior oferece vantagens por ser um método permanente de correção óptica, fornece uma imagem retiniana estável e provoca aniseiconia mínima. Além disso, a psedofacia oferece potencial para uma boa acuidade visual $^{(10)}$. No entanto complicações tem sido relatadas como implante de câmara posterior, tais como: severa reação inflamatória, glaucoma por bloqueio pupilar, formação de membrana secundária, deslocamento da lente e captura pupilar ${ }^{(10)}$.

A ausência de longos tempos de seguimento, o potencial para o desenvolvimento de complicações tardias induzidas pela lente e a não preditibilidade do erro refrativo pós-operatório em um olho que está em crescimento, são questões ainda inerentes ao tratamento da pseudofacia após catarata traumática em crianças.

\section{CONCLUSÂO}

O tratamento da catarata após trauma em crianças apresentou resultados visuais satisfatórios, sendo que nos olhos em que foram obtidas as acuidades visuais inicial e final, a melhora apresentada foi estatisticamente significante (teste de Wilcoxon $\mathrm{p}<0,001)$. O nível de significância porém foi maior para os olhos submetidos ao tratamento cirúrgico $(\mathrm{p}<0,001)$ quando comparados com os olhos submetidos ao tratamento clínico no serviço $(\mathrm{p}=0,043)$. 


\section{ABSTRACT}

Purpose: To study the pediatric cataract after trauma, it's relation to the kind of the trauma, the time elapsed between trauma and surgery and the correlation between corrected visual acuity and treatment. Methods: We reviewed the medical records of all patients who presented diagnosis of traumatic cataract between August 1988 and December 2001 at the Congenital Cataract Service of Federal University of São Paulo. Results: Sixty-six patients with diagnosis of pediatric cataract after trauma were studied, corresponding to an incidence of $4.80 \%$ of the total of cases attended in the service. Forty-seven $(71.22 \%)$ were male and $19(28.78 \%)$ were female. Forty-five $(53.03 \%)$ sustained a blunt trauma, $21(31.82 \%)$ a penetrating one, and 10 $(15.15 \%)$ were not classified. The mean time between the injury and the surgery was 7 years and 6 months. The main described postoperative complications were uveitis sequels in 13 patients $(21.12 \%)$ and posterior capsule opacification in $10(15.15 \%)$. The initial and final visual acuity were obtained in 30 eyes. The mean follow-up was of two years, ranging from 1 to 96 months. Conclusion: The improvement of visual acuity was statistically significant (Wilcoxon test $\mathrm{p}<0.001$ ), being more intense in the eyes submitted to surgical treatment $(\mathrm{p}<0.001)$ than in the eyes submitted to clinical treatment.

Keywords: Cataract extraction; Cataract/pathology; Visual acuity; Wounds and injuries; Wounds, penetrating; Pseudophakia

\section{REFERÊNCIAS}

1. Hoover LD, Smith H. Evaluation and management strategies for the pediatric eye trauma In: Shigleto JB, Hersh SP, Kenyon RK. Eye trauma. St Louis: Mosby; 1991. p.55-9.

2. Ben Ezra D, Cohen E, Rose L. Traumatic cataract in children: correction of aphakia by contact lens or intraocular lens. Am J Ophthalmol 1997;123:773-82.

3. Parks MM, Johnson DA, Reed GW. Long-term visual results and complications in children with aphakia. A function of cataract type. Ophthalmology 1993;100:826-40

4. Brar GS, Ram J, Pandav SS, Reddy GS, Singh U, Gupta A. Postoperative complications and visual results in uniocular pediatric traumatic cataract. Ophthalmic Surg Lasers 2001;32:233-8.

5. Scherer WJ, Hauber FA. Surgical outcomes in adult patients after repair of anterior segment trauma sustained during childhood. J Cataract Refract Surg 2001;27:256-60.

6. Bowman RJ, Yorston D, Wood M, Gilbert C, Foster A. Primary intraocular lens implantation for penetrating lens trauma in Africa. Ophthalmology 1998; 105:1770-4.

7. Eckstein M, Vijayakshmi P, Killedar M, Gilbert C, Foster A. Use of intraocular lenses in children with traumatic cataract in south India. $\mathrm{Br} \mathrm{J}$ Ophthalmol 1998;82:911-5.

8. Gradin D, Yorston D. Intraocular lens implantation for traumatic cataract in children in East Africa. J Cataract Refract Surg 2001;27:2017-25.

9. Churchill AJ, Noble BA, Etchells DE, George NJ. Factors affecting visua outcome in children following uniocular traumatic cataract. Eye 1995;9:285-91.

10. Koenig SB, Ruttum MS, Lewandowski MF, Schultz RO. Pseudophakia for traumatic cataracts in children. Ophthalmology 1993;100:1218-24.

11. Zaidman G, Ramirez T, Kaufman A, Palay D, Phillips R, Medow N. Successful surgical rehabilitation of children with traumatic corneal laceration and cataract. Ophthalmology 2001;108:338-42.

12. Moisseiev J, Segev F, Harizman N, Arazi T, Rotenstreich Y, Assia El. Primary cataract extraction and intraocular lens implantation in penetrating ocular trauma. Ophthalmology 2001;108:1099-103.

\section{Congresso Infernacional da Sociedade Brasileira de Offalmologia - SBO}

\section{9 a 12 de Junho de 2004 \\ Centro de Convenções Ribalta - RJ}

INFORMAÇÕES: Tel.: (21) 2557-7298

Fax: (21) 2205-2240 